\title{
Pulmonary Hypertension Associated with Postoperative Tetralogy of Fallot
}

\author{
Jun Yasuhara and Hiroyuki Yamagishi
}

\begin{abstract}
Keywords
Pulmonary artery · Major aortopulmonary collateral artery · Percutaneous pulmonary angioplasty · Pulmonary vascular bed · Segmental pulmonary hypertension
\end{abstract}

Pulmonary hypertension $(\mathrm{PH})$ is a frequent complication in patients with congenital heart disease (CHD), before or after cardiac surgery. According to the recent clinical classification, $\mathrm{PH}$ associated with $\mathrm{CHD}$ is categorized into subclasses depending on the pathogenesis: (1) Eisenmenger syndrome, (2) left-to-right shunts, (3) PH with coincidental CHD, and (4) postoperative $\mathrm{PH}$ [1]. Postoperative $\mathrm{PH}$ means that CHD was repaired, but $\mathrm{PH}$ either persists immediately after surgery or recurs/develops months or years after surgery, in the absence of significant postoperative hemodynamic lesions. The anatomical features are varied and the clinical phenotype is often aggressive [2-4].

We encountered many patients with $\mathrm{PH}$ associated with postoperative tetralogy of Fallot (TOF), especially those with major aortopulmonary collateral arteries (MAPCAs). The etiology of PH associated with TOF with or without MAPCAs is uncertain. During cardiovascular development, MAPCAs are formed to supply blood flow to the lungs through remnants of inter-segmental arteries (ISAs) as a result of abnormal regression of the sixth pharyngeal arch arteries (PAA) in the embryonic stages (Fig. 29.1). Maldevelopment of the sixth PAA also leads to

\footnotetext{
J. Yasuhara

Department of Pediatrics, Keio University School of Medicine, Shinjuku, Tokyo, Japan

H. Yamagishi $(\square)$

Division of Pediatric Cardiology, Department of Pediatrics,

Keio University School of Medicine, Tokyo, Japan

e-mail: hyamag@keio.jp
} 


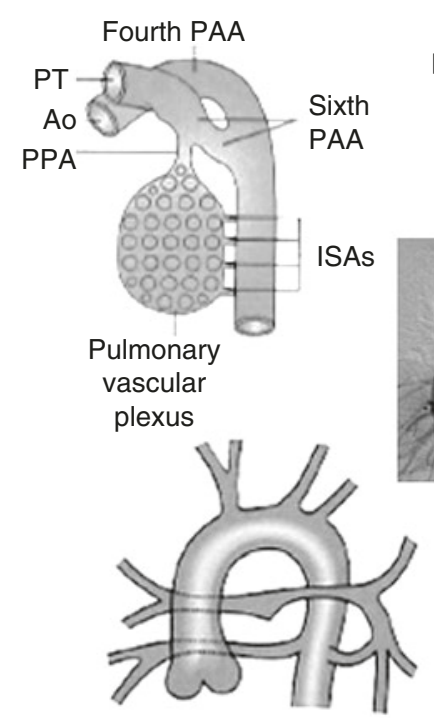

Bilateral PAs are MAPCA dependent Hypoplastic central PA is present ( $20 \%$ of TOF with Pat.)
Developing aortic and pulmonary artery system

Sixth PAA regresses, ISAs lead to MAPCAs

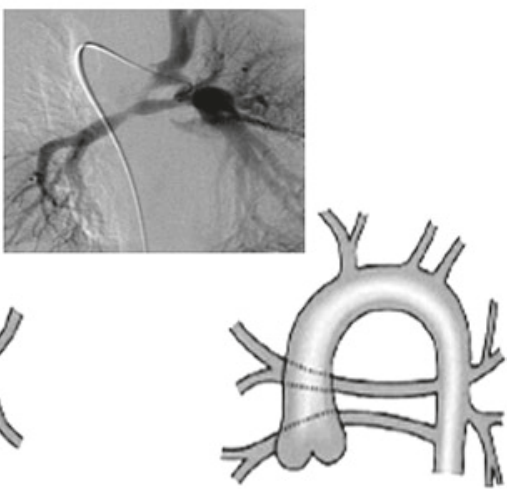

Bilateral PAs are MAPCA dependent No confluent central PA ( $10 \%$ of TOF with Pat.)

Fig. 29.1 Development of the aortic and pulmonary artery system, and the genesis of major aortopulmonary collateral arteries (MAPCAs). MAPCAs are formed to supply blood flow to the lungs through remnants of inter-segmental arteries (ISAs). Types of pulmonary blood supply in tetralogy of Fallot (TOF) with pulmonary atresia (Pat.) are illustrated with approximate frequencies. Pulmonary angiography of the patient is also shown. Ao aorta, $P A$ pulmonary artery, $P A A$ pharyngeal arch artery, $P P A$ peripheral pulmonary artery, $P T$ pulmonary trunk

hypoplasia or aplasia of the ductus arteriosus and peripheral pulmonary artery (PPA). The postoperative TOF, especially with MAPCAs, was implicated in hypoplasia of the lungs and pulmonary vasculature, thrombosis, and/or previously large systemic-to-pulmonary shunts. MAPCAs are connected to hypoplastic or segmentally unequal pulmonary vascular beds and branches, resulting in arborization abnormalities as observed in the pulmonary angiography (Fig. 29.1) [5]. We, therefore, speculate that inadequate pulmonary vascular beds due to segmentally unequal pulmonary blood flow, vascular spasm in the area of alveolar hypoventilation, and/ or residual peripheral pulmonary stenosis (PPS) may result in high pulmonary blood pressure in postoperative TOF with MAPCAs. The hemodynamic and clinical presentations of patients with $\mathrm{PH}$ associated with postoperative TOF and MAPCAs are sometimes similar to those in Eisenmenger syndrome.

To better recognize the PH associated with TOF, especially with MAPCAs, the updated subclass as "segmental PH associated with CHD" should be important. Our clinical experience suggests that PH-targeted therapy may be effective for some of such patients, especially combined with percutaneous pulmonary angioplasty for the repair of residual PPS. 


\section{References}

1. Simonneau G, Gatzoulis MA, Adatia I, et al. Updated clinical classification of pulmonary hypertension. J Am Coll Cardiol. 2013;62(25 Suppl):D34-41. https://doi.org/10.1016/j. jacc.2013.10.029.

2. Ivy DD, Abman SH, Barst RJ, et al. Pediatric pulmonary hypertension. J Am Coll Cardiol. 2013;62(25 Suppl):D117-26. https://doi.org/10.1016/j.jacc.2013.10.028.

3. D'Alto M, Mahadevan VS. Pulmonary arterial hypertension associated with congenital heart disease. Eur Respir Rev. 2012;21(126):328-37. https://doi.org/10.1183/09059180.00004712.

4. Marelli AJ, Mackie AS, Ionescu-Ittu R, et al. Congenital heart disease in the general population: changing prevalence and age distribution. Circulation. 2007;115(2):163-72.

5. Yasuhara J, Yamagishi H. Pulmonary arterial hypertension associated with tetralogy of Fallot. Int Heart J. 2015;56:S17-21. https://doi.org/10.1536/ihj.14-351.

Open Access This chapter is licensed under the terms of the Creative Commons Attribution 4.0 International License (http://creativecommons.org/licenses/by/4.0/), which permits use, sharing, adaptation, distribution and reproduction in any medium or format, as long as you give appropriate credit to the original author(s) and the source, provide a link to the Creative Commons license and indicate if changes were made.

The images or other third party material in this chapter are included in the chapter's Creative Commons license, unless indicated otherwise in a credit line to the material. If material is not included in the chapter's Creative Commons license and your intended use is not permitted by statutory regulation or exceeds the permitted use, you will need to obtain permission directly from the copyright holder. 